\title{
Major trauma mortality in rural and metropolitan NSW, 2009-2014: a retrospective analysis of trauma registry data
}

\author{
Michael M Dinh',2, Kate Curtis ${ }^{3}$, Rebecca J Mitchell ${ }^{4,5}$, Kendall J Bein ${ }^{2}$, Zsolt J Balogh ${ }^{6,7}$, Ian Seppelt ${ }^{8,9}$, Colin Deans ${ }^{10}$, \\ Rebecca Ivers ${ }^{11,12}$, Saartje Berendsen Russell ${ }^{2,13}$, Oran Rigby ${ }^{14}$
}

The known Trauma systems facilitate the timely treatment of major trauma patients at specialised centres, and this approach has reduced trauma-related mortality in Australia and overseas. Trauma in rural areas, however, has been associated with higher mortality.

The new Trauma system changes introduced to New South Wales since 2009 may be associated with a decline in crude and adjusted inpatient mortality after major trauma in rural and regional locations.

The implications Better transfer of rural patients, retrieval networks, and improved trauma care at major trauma centres may be benefiting severely injured patients from rural and regional locations in NSW.

I njury causes significant physical and psychological disability around the world, ${ }^{1,2}$ and cost-effective systems of care are important for optimising patient outcomes and recovery. Trauma systems facilitate the timely treatment of severely injured patients, and this approach has reduced mortality among trauma patients in several Australian states ${ }^{3,4}$ and overseas. ${ }^{5,6}$ A system for trauma care was introduced in New South Wales in 1991, and the first analyses of major trauma outcomes in NSW were conducted in 2012. ${ }^{7,8}$ It was found that a survival benefit was associated with definitive care at designated major trauma centres located in NSW metropolitan areas.

A number of factors may influence outcomes for trauma patients in rural and regional areas, particularly the sparse population density and the vast transport distances for many rural and regional trauma patients. Studies from other parts of Australia have indicated that rural trauma patients have poorer outcomes than metropolitan trauma patients, and it is recognised that road accidents in rural and remote locations are associated with higher risks of death and severe injury. ${ }^{9,10}$ The NSW Trauma Minimum Dataset now includes additional details on injuries, including those for a number of regional centres, ${ }^{11}$ allowing more detailed analyses of outcomes according to the geographic location of injuries incurred after 2009.

This change was particularly relevant because the revised NSW State Trauma Plan was implemented in 2009, formalising rural and regional referral networks for each of the seven adult major trauma centres. ${ }^{12}$ The aim of these networks was to facilitate the timely transfer of severely injured patients from sparsely populated rural and remote areas of NSW to major trauma centres in metropolitan areas along the east coast. We assessed and compared trends in crude and risk-adjusted mortality between 2009 and 2014 in the context of these changes, designed to improve trauma care and patient outcomes in NSW.

\section{Abstract}

Objective: To determine trends in crude and risk-adjusted mortality for major trauma patients injured in rural or metropolitan New South Wales, 2009-2014.

Design: A retrospective analysis of NSW statewide trauma registry data.

Participants: Adult patients (aged 16 years or more) who presented with major trauma (Injury Severity Scores greater than 15) to a NSW hospital during 2009-2014.

Main outcome measures: The main covariate of interest was geographic location of injury (metropolitan $v$ rural/regional areas). Inpatient mortality was analysed by multivariable logistic regression.

Results: Data for 11423 eligible patients were analysed. Inpatient mortality for those injured in metropolitan locations was $14.7 \%$ in 2009 and $16.1 \%$ in $2014(P=0.45)$. In rural locations, there was a statistically significant decline in in-hospital mortality over the study period, from $12.1 \%$ in 2009 to $8.7 \%$ in $2014(P=0.004)$. Risk-adjusted mortality for those injured in a rural location was lower in 2013 than during 2009, but remained stable for those injured in metropolitan locations.

Conclusion: Crude and risk-adjusted mortality after major trauma have remained stable in those injured in metropolitan areas of NSW between 2009 and 2014. The apparent downward trend in mortality associated with severe trauma in rural/regional locations requires further analysis.

\section{Methods}

NSW is the most populous Australian state, with a population of 7.5 million in 2014 and an area of $809000 \mathrm{~km}^{2}$. Around $70 \%$ of the population live in metropolitan areas on the eastern seaboard. ${ }^{13}$

We undertook a retrospective analysis of statewide trauma registry data. The statewide trauma registry was established and is maintained by the NSW Institute of Trauma and Injury Management; it receives data from seven adult major trauma centres and ten regional trauma centres. ${ }^{11}$ According to the standards of the American College of Surgeons, ${ }^{14}$ major trauma centres in NSW are equivalent to level 1 designated trauma centres (the top level), and regional trauma centres are equivalent to level 2 or 3 centres.

Adult patients (aged 16 years or more) were included in our analysis if they presented to a NSW hospital between 1 January 2009 and 31 December 2014, and their Injury Severity Score (ISS) was greater than 15 .

Trauma centres that did not submit data for the entire study period were excluded. Patients were also excluded if the postcode of the site 
where they were injured was unknown or outside NSW, as were those who were dead on arrival at hospital. Duplicate records for patients who had been transferred between hospitals were identified, and the second and subsequent records excluded if the referral hospital provided trauma data to the NSW Trauma Registry.

Basic demographic characteristics, the mechanism of injury, vital signs on arrival at hospital, length of stay in hospital, and in-hospital mortality were analysed. Injuries were classified according to the Abbreviated Injury Scale (AIS). The AIS codes injuries according to their anatomic location (head, neck, chest, abdomen, lower limb, upper limb, external) and assigns a severity score, ranging from 1 to 6 , according to the likelihood of death and disability. Severe injuries in this study were defined by AIS scores of 3 or more. ${ }^{15}$ The ISS, used to assess the overall severity of injury, was calculated by summing the squares of the AIS severity scores for the three most severely injured body regions. Major trauma was defined as an ISS greater than 15. Measures of hospital resource use included inter-hospital transfer, and the first major trauma procedures recorded by the referring hospital, ambulance or retrieval services, or trauma centre. The geographic location where the patient sustained their injury was identified by postcode, and categorised as metropolitan, inner regional, or outer regional/ remote according to the Australian Statistical Geography Standard Remoteness Structure. ${ }^{16}$ As the number of deaths in outer regional/remote regions was small, all non-metropolitan postcodes were merged as rural/regional for multivariable analyses.

The primary outcome for our study was in-hospital mortality after major trauma, analysed according to the geographic location of the injury.

\section{Statistical analyses}

Descriptive statistics for baseline characteristics and crude inhospital mortality are reported. Year-by-year differences and trends in crude in-hospital mortality were compared in $\chi^{2}$ and Cochran-Armitage linear trend tests. Risk-adjusted mortality trends were assessed in logistic regression models stratified by geographic location of the injury and adjusted for age, injury severity, intensive care unit admission, and year of admission. Variables were included in models according to the results of preceding univariate analyses $(P<0.1)$, or if they had been identified as influencing mortality risk in adult injury patients by previous studies. ${ }^{8}$ Adjusted odds ratios (ORs) for inpatient mortality in separate rural and metropolitan location regression models were plotted on a line chart to identify trend in ORs relative to the reference year, 2009. Multivariable adjusted trends were determined by changing the year predictor variable to a linear continuous variable for logistic regression modelling and non-parametric generalised additive models (GAM). Analyses were performed in SAS Enterprise Guide 6.1 (SAS Institute).

\section{Ethics approval}

Approval was obtained from the NSW Population Health Services and Research Ethics Committee (reference, 2015/04/036).

\section{Results}

\section{Study population}

A total of 18652 patients were identified in the trauma registry data for the period 2009-2014. After the exclusions described in the Methods, data for 11423 adult patients with an ISS greater than 15 from seven adult major trauma centres and three regional trauma centres were analysed (Appendix).

Box 1 compares the demographic characteristics of rural/regional (combined inner, outer regional and remote injury locations) and metropolitan major trauma patients, and the clinical characteristics of their injuries. With respect to location of injury, 8878 patients $(77.7 \%)$ were in metropolitan locations, $1855(16.2 \%)$ in inner regional locations, 601 (5.3\%) in outer regional locations, and 89 $(0.8 \%)$ in remote locations. The mean age of the patients was 53.5 years (SD, 23.1); $71.9 \%$ of the patients were men. The most common mechanisms of injury were falls $(44.3 \%)$, road trauma $(37.6 \%)$ and blunt assault (5.9\%); penetrating injuries accounted for $3.6 \%$ of all cases.

The distribution of ages of rural/regional trauma patients, compared with metropolitan patients, was shifted to younger age groups. The proportions of head, chest, spinal, and lower limb severe injuries were higher for rural/regional trauma patients, consistent with the higher proportion of road trauma cases in this patient population (Box 1).

\section{Inpatient major trauma mortality}

Almost half of major trauma inpatient deaths (46.6\%) occurred within 24 hours of admission, a further $30 \%$ occurred during the first week of admission, and 23.4\% occurred after the second week of admission. The overall inpatient mortality rate for major trauma was $14.1 \%$ in 2009 and $14.5 \%$ in 2014, with no significant trend (Cochran-Armitage test, $P=0.66$ ). There was no difference in overall inpatient mortality between admissions to major trauma and non-major trauma centres $(14.0 \% v 13.0 \% ; P=0.47)$. For those injured in metropolitan locations, inpatient mortality was $14.7 \%$ in 2009 and 16.1\% in 2014 (Cochran-Armitage test, $P=0.45$ ). Major trauma in rural/regional locations was associated with a statistically significant decrease in in-hospital mortality over this period, from $12.1 \%$ in 2009 to $8.7 \%$ in 2014 (Cochran-Armitage test, $P=0.004$ ). When rural/regional location was further stratified, major trauma mortality in inner regional locations decreased from $12.7 \%$ in 2009 to $10.4 \%$ in $2014(P=0.07)$; for outer regional/remote locations, it decreased from $10.3 \%$ in 2009 to $4.0 \%$ in $2014(P=0.005)$.

Box 2 shows the trend in risk-adjusted mortality for rural/regional and metropolitan injuries, compared with the reference year 2009. The adjusted OR for in-hospital mortality associated with rural/ regional injuries was lower in 2013 (OR, 0.5; 95\% CI, 0.3-0.8) and 2014 (OR, 0.6; 95\% CI, 0.4-1.0) than in 2009. Mortality among rural patients declined by an average of $12 \%$ per year when year was analysed as a linear predictor (OR, 0.88; 95\% CI, 0.81-0.96; $P=0.004)$; non-parametric GAM analysis indicated that this decline was statistically significant (multivariable GAM, $P=0.007)$. In contrast, there was no trend in risk-adjusted mortality for injuries in metropolitan locations (multivariable GAM, $P=0.25)$.

\section{Mode of arrival and inter-hospital transfer}

For the 2292 major trauma patients injured in a rural location and admitted to a major trauma centre, there was no change between 2009 and 2014 in the proportion who arrived directly (not transferred from another hospital) at a major trauma centre from a rural location (38.7\% v 36.7\%; Cochran-Armitage test, $P=0.96$ ). There was an increase in the proportion who arrived at a major trauma centre by ambulance from a rural location $(26.2 \% v 60.6 \%$; $P<0.001)$, and a decline in the median time from injury to first trauma procedure from $276 \mathrm{~min}$ (interquartile range [IQR], $104-1420)$ in 2010 to $95 \mathrm{~min}(\mathrm{IQR}, 48-495)$ in $2014(P<0.001)$. 
1 Baseline demographic data, clinical characteristics, and in-hospital mortality for 11423 major trauma patients (Injury Severity Score > 15), New South Wales Trauma Registry, 2009-2014, by geographic location of injury

\begin{tabular}{cccc}
\multicolumn{4}{c}{ Metropolitan Rural/regional } \\
NSW & NSW & $P$ \\
\hline
\end{tabular}

Total number $\quad 8878 \quad 2545$

of patients

Age group

$16-24$ years

$1191 \quad 13.4 \% \quad 473 \quad 18.6 \%$

25-44 years

$2108 \quad 23.7 \% \quad 735 \quad 28.9 \%$

45-64 years

$2091 \quad 23.6 \% \quad 678 \quad 26.7 \%$

$64-84$ years

$2441 \quad 27.5 \% \quad 565 \quad 22.2 \%$

$>84$ years

$1047 \quad 11.8 \% 94$

$3.7 \%$

Sex

$\begin{array}{lllll}\text { Men } & 6294 & 70.9 \% & 1920 & 75.4 \%\end{array}$

Mechanism of injury

Road trauma

Falls

Penetrating trauma

Blunt assaults

Burns

Other

Injury Severity Score (ISS)

$$
\begin{aligned}
& 15-24 \\
& 25-49
\end{aligned}
$$

$\geq 50$

Severe injury site (AIS > 2)

Head

Chest

Abdomen

Spine/vertebral

column

\section{Upper limb}

Lower limb

External

$2994 \quad 33.7 \% \quad 1298 \quad 51.0 \%$

$4276 \quad 48.2 \% \quad 713 \quad 28.0 \%$

$347 \quad 3.9 \% \quad 72 \quad 2.8 \%$

$527 \quad 5.9 \% \quad 150 \quad 5.9 \%$

$180 \quad 2.0 \% \quad 41 \quad 1.6 \%$

$554 \quad 6.3 \% \quad 269 \quad 10.6 \%$

$5119 \quad 57.7 \% \quad 1428 \quad 56.1 \%$

$3518 \quad 39.6 \% \quad 1036 \quad 40.7 \%$

$241 \quad 2.7 \% \quad 81 \quad 3.2 \%$

$5128 \quad 57.8 \% \quad 1266$

$3028 \quad 34.1 \% \quad 1080 \quad 42.4 \%<0.001$

$49.7 \%<0.001$

$\begin{array}{lllll}855 & 9.6 \% & 275 & 10.8 \% & 0.08\end{array}$

$997 \quad 11.2 \% \quad 413 \quad 16.2 \%<0.001$

$\begin{array}{lllll}104 & 1.2 \% & 48 & 1.9 \% & 0.005\end{array}$

$1342 \quad 15.1 \% \quad 496 \quad 19.5 \%<0.001$

$\begin{array}{lllll}143 & 1.6 \% & 32 & 1.2 \% & 0.20\end{array}$

Mode of arrival to initial hospital

Ambulance

Helicopter

Fixed wing aircraft

Private vehicle

Other

Inpatient deaths

\begin{tabular}{lllllc}
$2009(N=1790)$ & 209 & $14.7 \%$ & 45 & $12.1 \%$ & 0.19 \\
$2010(N=1749)$ & 207 & $15.4 \%$ & 49 & $12.1 \%$ & 0.10 \\
$2011(N=1808)$ & 216 & $15.4 \%$ & 39 & $9.6 \%$ & 0.003 \\
$2012(N=1895)$ & 206 & $14.5 \%$ & 41 & $8.7 \%$ & 0.002 \\
$2013(N=2062)$ & 250 & $15.2 \%$ & 26 & $6.2 \%$ & $<0.001$ \\
$2014(N=2119)$ & 266 & $16.1 \%$ & 41 & $8.7 \%$ & $<0.001$ \\
\hline
\end{tabular}

AIS = Abbreviated Injury Scale score. $N=$ Number of major trauma cases for year.

\section{Discussion}

We found that overall inpatient mortality after major trauma remained steady between 2009 and 2014, but crude mortality declined among patients severely injured in rural and regional NSW. There was also a reduction in risk-adjusted mortality associated with rural location of injury. Comparison of data on crude inpatient mortality identified that this trend was most marked for injuries incurred in outer regional and remote locations.

These findings could be explained by a number of factors. Firstly, the establishment of trauma referral networks may have resulted in more efficient transfers between rural facilities and major trauma centres. We found that the time to first trauma procedure for patients from rural areas had declined since 2009. The NSW Ambulance Service trauma bypass protocol (protocol T1), revised in 2008, allowed for an increase in acceptable transfer time between the scene of the injury and arrival at the nearest designated trauma centre from 30 minutes to one hour. ${ }^{17}$ In addition, the introduction of the Rapid Launch Trauma Coordinator function in the NSW Ambulance Aeromedical Control Centre aimed to improve the early identification of major trauma incidents by monitoring emergency 000 calls to ensure that specialist prehospital resources are activated as early as possible. Both factors may have contributed to the steady increase in direct ambulance arrivals at major trauma centres from rural locations between 2009 and 2014. This has occurred although the proportion of interhospital transfers from rural and regional hospitals to major trauma centres was unchanged. It is unclear whether direct or secondary transfer from a rural hospital or direct transport to a major trauma centre is associated with improved survival. A 2011 systematic review of 36 observational studies found no difference, although most investigations were subject to potential referral bias, in that they excluded deaths from the data for referring hospitals. ${ }^{18}$

Secondly, improved outcomes for severely injured rural patients may have resulted from improved clinical care in regional trauma centres and rural referral centres. A core mission of the NSW Institute for Trauma and Injury Management over the past decade has been to coordinate and improve access to clinical expertise and education resources in these centres. ${ }^{12}$ This approach included employing regional trauma nurse coordinators, which achieved improved education, case management, data collection and audit capabilities. An American study of 18 rural level 3 and 4 trauma centres found that a rural trauma education course alone reduced the time to transfer of severely injured patients. ${ }^{19}$

Thirdly, the reduction in major trauma mortality in outer regional and remote locations underscores the importance of road safety initiatives for preventing deaths and critical injuries. The reductions reported here mirror the $11 \%$ drop in the rate of road accident deaths (per 100000 population) in very remote locations between 2008 and 2012, compared with reductions of $0.7 \%$ in inner regional and $0.9 \%$ in metropolitan areas. ${ }^{20}$ The National Road Safety Strategy highlights the need to prioritise improving high risk rural and urban roads, vehicle safety standards, and safety for vulnerable road users. ${ }^{20}$ Nevertheless, rural road deaths continue to occur at two to three times the national average because of the longer travel distances, higher vehicle speed zones, and the greater likelihood of head-on collisions and vehicle rollovers than in more urban locations. ${ }^{21}$ This problem requires further attention and investment from all levels of government. 
2 Risk-adjusted odds ratios for mortality following major trauma, New South Wales, 2009-2014, stratified by geographic location of injury*

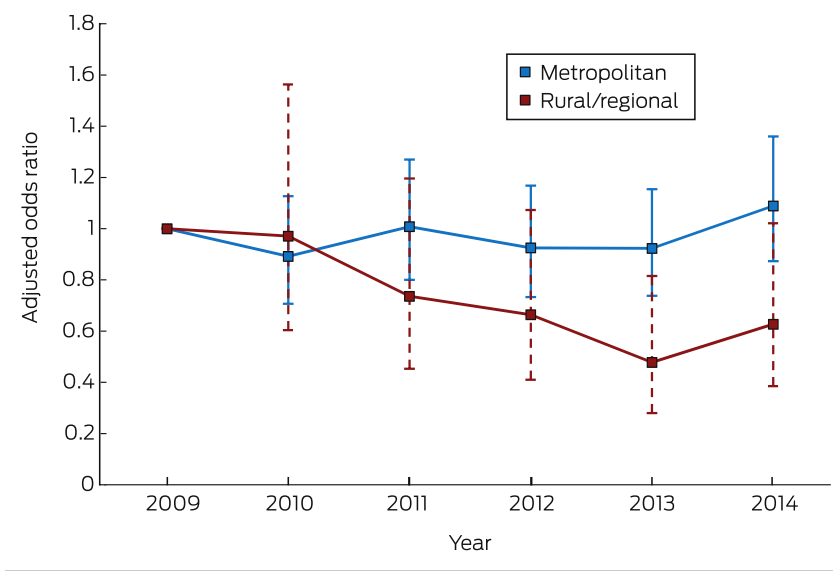

* The reference year is 2009. Estimates and 95\% confidence intervals can be compared with the reference year, but not between rural/regional and metropolitan locations.

The lack of overall improvement in major trauma mortality in metropolitan NSW since the 2012 study by Curtis and colleagues ${ }^{8}$ is concerning. These authors had reported that overall mortality for NSW patients (rural and metropolitan) with an ISS greater than 15 had declined from $15.0 \%$ in 2003 to $12.9 \%$ in 2007. Mortality among trauma patients appears to have returned to around $15 \%$, highlighting the ongoing need for quality improvements in the major trauma system in NSW, including regionalisation of trauma centres ${ }^{22}$ and models of care that sustainably manage the growing proportion of older major trauma patients. ${ }^{23}$ We found that the overall mortality of patients with an ISS greater than 12 during 2013-14 (all centres submitted data during this period for patients with an ISS over 12) was $11.1 \%$, similar to the $11 \%$ reported by the Victorian population-based trauma registry for 2013-14. ${ }^{24}$

It remains to be seen whether risk-adjusted mortality associated with trauma in rural/regional areas continues to fall. Despite the downward trend in Box 2, the decrease was statistically significant only for 2013. The relatively small sample of patients in the rural/ regional cohort may explain the lack of statistical significance for other years, as confidence limits for estimates were narrower when rural and regional case numbers were higher. Further analyses that include all years with complete data, including physiological data from rural/regional trauma centres, are needed to assess these trends, as is further risk adjustment modelling, consistent with analyses by other trauma registries. ${ }^{24}$
We also acknowledge other limitations of our study. Firstly, seven regional trauma centres did not submit data to the statewide trauma registry until after 2012, and were therefore not included in our study. Data for people who died at the scene of an injury were also excluded, and this may have biased our assessment of rural trauma mortality. Secondly, formal risk adjustment of inpatient mortality according to physiologic parameters was not possible, as vital signs data were not recorded for almost $30 \%$ of all cases in the trauma registry. Risk adjustment is important in order to correct for possible differences between locations and facilities in patient characteristics, as well as in mechanisms of injury, age, and physiological parameters. We utilised admission to an intensive care unit as a proxy marker for physiological abnormality, but the risk adjustment models reported here are not comparable with those of the Australian Trauma Registry or with similar studies used to benchmark performance of trauma systems across different locations and facilities. ${ }^{25}$ Finally, the period 2009-2012 saw the transition from individual hospital-based trauma registries to a uniform statewide registry designed to streamline data collection, auditing and research across all designated trauma centres. Some of the problems of incomplete data (ie, trauma centres unable to submit data for the full period) may be explained by this transition period.

As the purpose of our study was to evaluate in-hospital mortality and overall trauma system performance and trauma centre care, we analysed only patients who survived to hospital presentation and excluded those who died at the scene of the injury or were declared dead on arrival. These deaths, however, would be reflected in the overall road toll statistics, which have also documented a decline in mortality in NSW. ${ }^{20}$ Taken together, the results of our analysis and the declining rural road toll favour our interpretation that overall trauma mortality has declined markedly in rural areas since 2009.

In conclusion, crude and risk-adjusted inpatient mortality associated with major trauma has remained stable for people injured in metropolitan areas of NSW since 2009. The reduction in risk-adjusted mortality in rural and regional NSW is encouraging, but further analyses will be appropriate when data from other NSW regional trauma centres become available.

Acknowledgements: This research was funded by the NSW Institute of Trauma and Injury Management (ITIM). The authors thank the NSW ITIM for providing access to the NSW Trauma Registry. The reported conclusions are those of the authors, and views expressed are not necessarily those of the funding agency. Kate Curtis is partly funded by an NHMRC Translation of Research into Practice Fellowship (GNT 1067639).

Competing interests: No relevant disclosures.

Received 8 Apr 2016, accepted 28 July 2016.

(c) 2016 AMPCo Pty Ltd. Produced with Elsevier B.V. All rights reserved.
1 GBD 2013 Mortality and Causes of Death Collaborators. Global, regional, and national age-sex specific all-cause and cause-specific mortality for 240 causes of death, 1990-2013: a systematic analysis for the Global Burden of Disease Study 2013. Lancet 2015; 385: 117-171.

2 Haagsma JA, Graetz N, Bolliger I, et al. The global burden of injury: incidence, mortality, disability-adjusted life years and time trends from the Global Burden of Disease study 2013. Inj Prev 2016; 22: 3-18.

3 Brennan PW, Everest ER, Griggs WM, et al. Risk of death among cases attending South Australian major trauma services after severe trauma: the first 4 years of operation of a state trauma system. $J$ Trauma 2002; 53: 333-339.
4 Cameron PA, Gabbe BJ, Cooper DJ, et al. A statewide system of trauma care in Victoria: effect on patient survival. Med J Aust 2008; 189: 546-550. https://www. mja.com.au/journal/2008/189/10/statewide-systemtrauma-care-victoria-effect-patient-survival

5 Twijnstra MJ, Moons KGM, Simmermacher RKJ, Leenen LPH. Regional trauma system reduces mortality and changes admission rates: a before and after study. Ann Surg 2010; 251: 339-343.

6 Celso B, Tepas J, Langland-Orban B, et al. A systematic review and meta-analysis comparing outcome of severely injured patients treated in trauma centers following the establishment of trauma systems. J Trauma 2006; 60: 371-378.
7 Mitchell RJ, Curtis K, Chong S, et al. Comparative analysis of trends in paediatric trauma outcomes in New South Wales, Australia. Injury 2013; 44: 97-103.

8 Curtis KA, Mitchell RJ, Chong SS, et al. Injury trends and mortality in adult patients with major trauma in New South Wales. Med J Aust 2012; 197: 233-237. https://www.mja. com.au/journal/2012/197/4/injury-trends-and-mortalityadult-patients-major-trauma-new-south-wales

9 Fatovich DM, Phillips M, Langford SA, Jacobs IG. A comparison of metropolitan vs rural major trauma in Western Australia. Resuscitation 2011; 82: 886-890.

10 Chen HY, Senserrick T, Martiniuk AL, et al. Fatal crash trends for Australian young drivers 1997-2007: 
geographic and socioeconomic differentials. J Safety Res 2010; 41: 123-128.

11 NSW Institute of Trauma and Injury Management Trauma data requirements in NSW [website]. https:// www.aci.health.nsw.gov.au/get-involved/institute-oftrauma-and-injury-management/Data/nsw_trauma_ registry system/trauma data requirements in nsw (accessed Apr 2016).

12 NSW Institute of Trauma and Injury Management. History of the NSW trauma system [website]. http:// www.aci.health.nsw.gov.au/get-involved/institute-oftrauma-and-injury-management/clinical/trauma system/history_of_the_nsw_trauma_system (accessed Mar 2016).

13 Australian Bureau of Statistics. 3218.0. Regional population growth 2013-14. Updated March 2015. http://www.abs.gov.au/AUSSTATS/abs@.nsf/ DetailsPage/3218.02013-14?0penDocument (accessed Apr 2016).

14 Royal Australasian College of Surgeons. The Australasian trauma verification program manual. Melbourne: Royal Australasian College of Surgeons, 2009. https://www. surgeons.org/media/309244/2009-08-04_Trauma Verification_Manual.pdf (accessed Apr 2016).
15 Association for the Advancement of Automotive Medicine. The Abbreviated Injury Scale 2005. Update 2008. Barrington, Ill: Association for the Advancement of Automotive Medicine, 2008.

16 Australian Bureau of Statistics. The Australian Statistical Geography Standard Remoteness Structure [website]. Updated June 2014. http://www.abs.gov.au/websitedbs/ d3310114.nsf/home/remoteness +structure\#Anchor2f (accessed Mar 2016).

17 Dinh MM, Oliver M, Bein KJ, et al. Performance of the New South Wales Ambulance Service major trauma transport protocol ( $\mathrm{Tl})$ at an inner city trauma centre. Emerg Med Australas 2012; 24: 401-407.

18 Hill AD, Fowler RA, Nathens AB. Impact of interhospital transfer on outcomes for trauma patients: a systematic review. J Trauma 2011; 71: 1885-1900

19 Kappel DA, Rossi DC, Polack EP, et al. Does the rural trauma team development course shorten the interval from trauma patient arrival to decision to transfer? J Trauma 2011; 70: 315-319.

20 Bureau of Infrastructure, Transport and Regional Economics. Impact of road trauma and measures to improve outcomes (Research report 140). Canberra:
BITRE, 2014. https://bitre.gov.au/publications/2014/ files/report_140.pdf (accessed Apr 2016).

21 Mitchell R, Chong S. Comparison of injury-related hospitalised morbidity and mortality in urban and rural areas in Australia. Rural Remote Health 2010; 10: 1326

22 Weinberg JA, Fabian TC. Does volume affect outcome with severe trauma? Adv Surg 2015; 49: 235-245.

23 Walter $\mathrm{E}$, Curtis $\mathrm{K}$. The role and impact of the specialist trauma nurse: an integrative review. J Trauma Nurs 2015; 22: 153-169.

24 Monash University, Victorian State Trauma Outcome Registry and Monitoring Group. Victorian State Trauma System and Registry. 1 July 2013 to 30 June 2014: summary report. Melbourne: Department of Health and Human Services, 2015. https://www2. health.vic.gov.au/Api/downloadmedia/\%7B1721A8 CB-4D4D-4CB1-ACA9-A415F6487FA6\%7D (accessed Apr 2016).

25 Alfred Health. Caring for the severely injured in Australia: inaugural report of the Australian Trauma Registry. 2010 to 2012. Melbourne: Alfred Health, 2014. http://ntri.org. au/img/aust-trauma-registry-inaugural-report.pdf (accessed Apr 2016) 